\title{
Correction: Enhanced tumorigenicity by mitochondrial DNA mild mutations
}

\author{
Alberto Cruz-Bermúdez ${ }^{1,2,3}$, Carmen G. Vallejo ${ }^{1}$, Ramiro J. Vicente-Blanco ${ }^{1,2}$, María \\ Esther Gallardo ${ }^{1,2,3}$, Miguel Ángel Fernández-Moreno ${ }^{1,2,3}$, Miguel Quintanilla ${ }^{1,4}$ and \\ Rafael Garesse ${ }^{1,2,3}$ \\ 1nstituto de Investigaciones Biomédicas "Alberto Sols", Consejo Superior de Investigaciones Científicas (CSIC), Universidad \\ Autónoma de Madrid (UAM), Madrid, Spain \\ ${ }^{2}$ Departamento de Bioquímica and Centro de Investigación Biomédica en Red en Enfermedades Raras (CIBERER), Facultad \\ de Medicina, UAM, Madrid, Spain \\ ${ }^{3}$ Instituto de Investigación Sanitaria Hospital 12 de Octubre (i+12), Madrid, Spain \\ ${ }^{4}$ Instituto de Investigación Hospital Universitario La Paz (IdiPAZ), Madrid, Spain
}

Published: March 17, 2020

Copyright: Cruz-Bermúdez et al. This is an open-access article distributed under the terms of the Creative Commons Attribution License 3.0 (CC BY 3.0), which permits unrestricted use, distribution, and reproduction in any medium, provided the original author and source are credited.

This article has been corrected: The Acknowledgements section has been updated as follows:

\section{ACKNOWLEDGMENTS}

This work was supported by the "Instituto de Salud Carlos III" [PI 10/0703 and PI13/00556 to R.G and PI 04/1001 to MAFM]; "Comunidad Autónoma de Madrid" [S2010/BMD-2402 to R.G and S2010/BMD-2359 to MQ); "Fundación Mutua Madrileña" [10.04.02.0064 to MAFM], the Spanish Ministry of Economy and Competitiveness (grant SAF2013-46183-R to MQ) and FEDER funds from the E.U. to R.G. M.E.G. is a staff scientist at the Centro de Investigación Biomédica en Red en Enfermedades Raras (CIBERER), Spain. Flow cytometry analyses were carried out at the "Servicio Interdepartamental de Investigacion" (SIdI) with the excellent technical assistance of Laura Molero Martín.

Original article: Oncotarget. 2015; 6:13628-13643. https://doi.org/10.18632/oncotarget.3698 